\title{
Electromagnetic effects as a new source of information on the space-time evolution of heavy ion collisions
}

\author{
Nikolaos Davis ${ }^{1, a}$, Andrzej Rybicki ${ }^{1}$, and Antoni Szczurek ${ }^{1,2}$ \\ ${ }^{1} \mathrm{H}$. Niewodniczański Institute of Nuclear Physics, Polish Academy of Sciences, Radzikowskiego 152, 31 - \\ 342 Kraków, Poland \\ ${ }^{2}$ University of Rzeszów, Rejtana 16, 35-959 Rzeszów, Poland
}

\begin{abstract}
We review our studies of spectator-induced electromagnetic (EM) effects on charged pion emission in ultrarelativistic heavy ion collisions. These effects are found to consist in the electromagnetic charge splitting of pion directed flow as well as very large distortions in spectra and ratios of produced charged particles. As it emerges from our analysis, they offer sensitivity to the actual distance, $d_{E}$, between the pion formation zone at freeze-out and the spectator matter. As a result, this offers a new possibility of studying the space-time evolution of dense and hot matter created in the course of the collision. Having established that $d_{E}$ traces the longitudinal evolution of the system and therefore rapidly decreases as a function of pion rapidity, we investigate the latter finding in view of pion feed-over from intermediate resonance production. As a result, we obtain a first estimate of the pion decoupling time from EM effects which we compare to existing HBT data. We conclude that spectator-induced EM interactions can serve as a new tool for studying the space-time characteristics and longitudinal evolution of the system. We discuss the future perspectives for this activity on the basis of existing and future data from the NA61/SHINE experiment.
\end{abstract}

\section{Introduction}

In the present work, we review various aspects of the final state electromagnetic (EM) interactions between the charged pions produced during an ultrarelativistic heavy-ion collision and the positively charged nuclear remnant ("spectator system") of the colliding nuclei, which does not participate directly in the collision. We argue that the study of the induced EM effects provides a useful new source of information on the space-time evolution of the system, valid in a broad range of collision centrality.

\section{EM effects in heavy ion collisions}

It is well established that charged spectator systems in non-central heavy ion collisions are the source of EM fields of considerable strength, which have a significant effect on the final state distributions of positively and negatively charged pions produced during freeze-out, as well as influence a variety of other observable quantities, such as the $\pi^{+} / \pi^{-}$ratios and directed flow as a function of the longitudinal

ae-mail: nikolaos.davis@ifj.edu.pl 
momentum of pions $[1,2]$. Below, we will review specific aspects of these phenomena, focusing mainly on the applicability of EM effects as a new source of information on the space-time evolution of the system.

The effect of spectator EM fields on the phase-space distributions of produced pions is evident in Fig. 1. The measurements were performed by the NA49 experiment (CERN SPS) in peripheral and intermediate centrality $\mathrm{Pb}+\mathrm{Pb}$ collisions at $\sqrt{s_{N N}}=17.3 \mathrm{GeV}$. In panel $1 \mathrm{a}(1 \mathrm{~b})$, we see that the invariant double differential spectra of positive (negative) pions reveal a suppression (enhancement) of produced pions for low values of transverse momentum, $p_{T}$, in the vicinity of longitudinal momentum corresponding to pions comoving with the spectator system, $x_{F}=0.15=m_{\pi} / m_{p}$. This isospin symmetry-breaking distortion of pion spectra becomes even more apparent if we examine the $\pi^{+} / \pi^{-}$ ratios as a function of transverse momentum for $x_{F}=0.15$, Fig. 1(c). We notice that the distortion of ratios is very similar for peripheral and intermediate centrality collisions. The weak centrality dependence of this effect may at first seem surprising, however it can be explained as a consequence of the reduced charge of spectators partially compensated by the decrease of impact parameter $b$ with increasing collision centrality. Another noteworthy effect observed in NA49 data is the significant enhancement, for low $p_{T}$ values, of the ratio $R$ of invariant particle densities of positive and negative pions produced in $\mathrm{Pb}+\mathrm{Pb}$ collisions, compared to the same quantity in $\mathrm{p}+\mathrm{p}$ collisions, as a function of longitudinal pion momenta, Fig. 2. As can be seen, the effect actually consists of a strong enhancement for $\pi^{-}$alone and a suppression for $\pi^{+}$, indicating a strong isospin symmetry-breaking effect, possibly of largely EM origin.

The influence of EM effects can also be detected in the behavior of collective azimuthal anisotropies (directed flow) of emitted charged pions with regards to the reaction plane, as detailed in [4]. In Fig. 3, directed flow $v_{1}$ is plotted as a function of the ratio of pion to beam rapidity in the c.m.s., for peripheral collisions recorded by the STAR and WA98 experiments.

By combining the results of the above measurements, we can obtain information on the dependence of EM effects of spectators on the rapidity of pions produced during freeze-out. Results were interpreted by means of a simplified one-point pion emission model, where the propagation of charged pions through the spectator EM field was numerically computed using classical relativistic equations of motion [1], while the spectators were represented as Lorentz-contracted homogeneously charged spheres, Fig. 4(a). The crucial free parameter in this model was the "pion emission distance" $d_{E}$ between the pion emission point and the spectator system. As an example, Fig. 4(c) shows Monte Carlo results for the $\pi^{+} / \pi^{-}$ratio for a specific choice of $d_{E}$. It is evident that by fine-tuning $d_{E}$, we can obtain a fairly good agreement with the measured NA49 ratios, Fig. 4(b). Similarly, Fig. 3(b) shows the values of $d_{E}$ furnished by the MC for different pion rapidities, $y / y_{\text {beam }}$.

\section{Sensitivity of EM effects to the space-time evolution of the system}

The final state distributions of pions measured in the detector are a direct result of the distortions induced by the spectator EM field on the trajectories of pions produced during the freeze-out. It is therefore not surprising that final state spectra appear sensitive to the space-time evolution of the particle production process. As was demonstrated in $[1,2]$, the general features of the distortions of pion spectra, as well as charge-dependent effects on directed flow, are sensitive to the distance $d_{E}$ between the longitudinal position of the pion formation zone at freeze-out and the longitudinal position of the spectator system at the same moment in time (in the c.m.s. reference frame), as schematically illustrated in Fig. 5 (a). Results furnished by our one-point pion emission model simulations indicate that the $\pi^{+} / \pi^{-}$ratio distortions shown in Fig. 4 correspond to a pion formation zone of pions comoving with spectators $\left(y \approx y_{\text {beam }}\right)$ being about $d_{E} \approx 0.75 \mathrm{fm}$ behind the spectator system in the longitudinal 

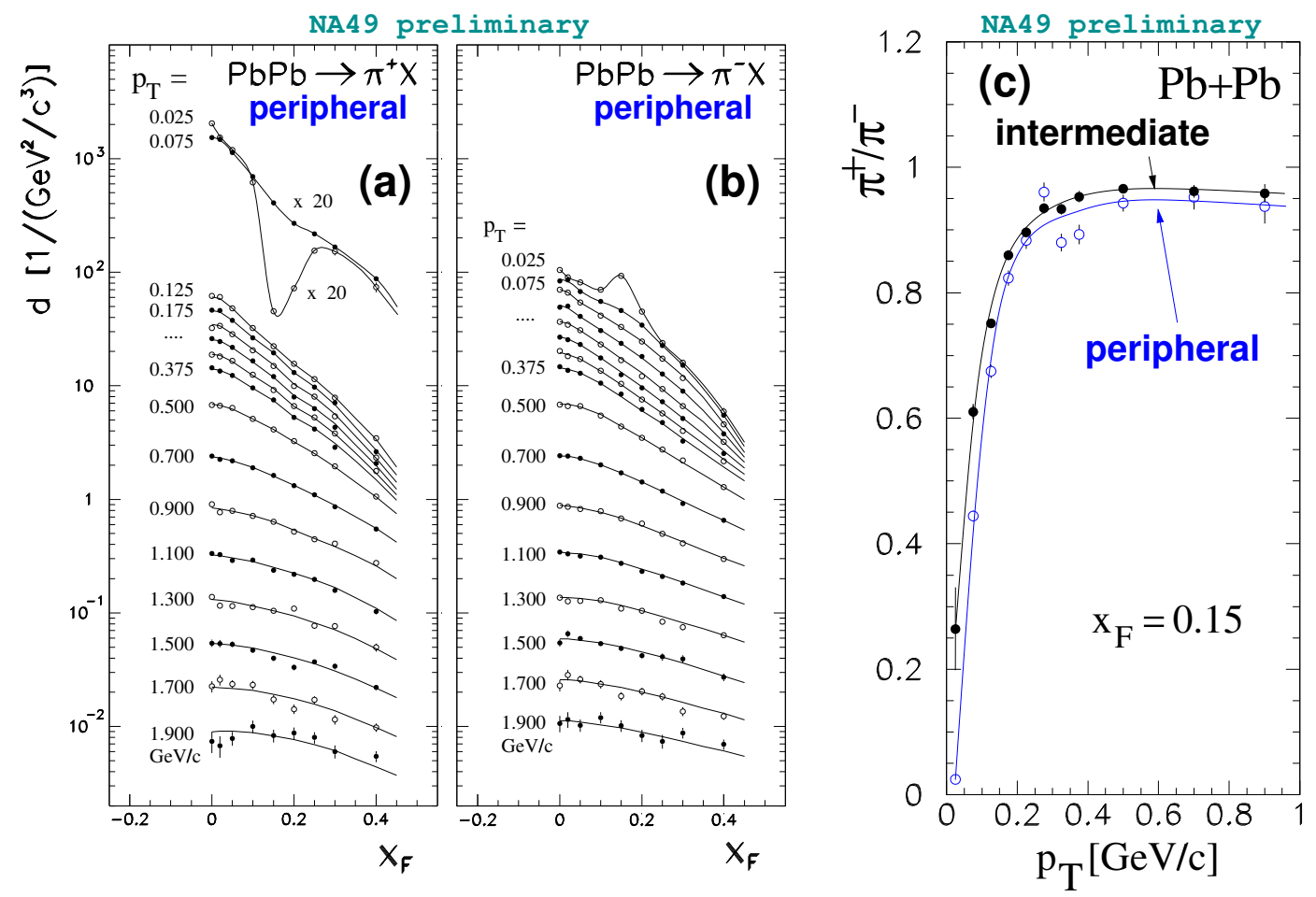

Figure 1. Spectator-induced EM effects on final state charged $\pi$ meson spectra and $\pi^{+} / \pi^{-}$ratios in $\mathrm{Pb}+\mathrm{Pb}$ collisions at $\sqrt{s_{N N}}=17.3 \mathrm{GeV}$. Double differential $d=E \frac{d^{3} N}{d p^{3}}$ distributions of (a) positive and (b) negative pions drawn as a function of $x_{F}=\frac{2 p_{L}}{\sqrt{s_{N N}}}$ and transverse momentum $p_{T}$ (both quantities in the collision c.m.s.), and (c) ratio of $\pi^{+}$over $\pi^{-}$distributions at $x_{F}=0.15$. Plot from [3].

direction. Similarly, the charge-splitting of directed flow observed in STAR Collaboration $\mathrm{Au}+\mathrm{Au}$ collisions at $\sqrt{s_{N N}}=7.7 \mathrm{GeV}$ [5], Fig. 3(a), corresponds to approximately $d_{E} \approx 3 \mathrm{fm}$ for pions produced at low c.m.s. rapidities, whereas WA98 experiment [7] measurements of very large $\pi^{+}$directed flow seen in target vicinity in $\mathrm{Pb}+\mathrm{Pb}$ collisions at the top SPS energy, Fig. 3(b), would suggest $d_{E} \approx 0.5 \mathrm{fm}$ at still higher rapidities. In Fig. $5(\mathrm{~d})$, these results are summarized, and we clearly notice a trend of decreasing $d_{E}$ with increasing pion rapidity.

In order to further investigate this result, a Monte-Carlo model was constructed incorporating existing knowledge about the production and decay properties of baryonic $(\Delta)$ and mesonic $(\rho)$ resonances, as described in [9]. In Fig. 5(b), a sketch of the model is given where the simplifying assumption is made that resonances are produced at the interaction point instantly, that is at $\tau=0$. As is evident from Fig. 5(d), this fails to reproduce the observed values of $d_{E}$ obtained from EM effects. In Fig. 5(c), a different model is sketched, for which the intermediate system is given a proper lifetime $\tau$, which can be adjusted to fit the observed $d_{E}$ curve. This suggests that quantitative information can be obtained on the spacetime properties and evolution of the hot and dense matter produced in a collision by studying spectator-induced EM effects. In Fig. 5(e), we explore the range of uncertainties introduced by incomplete knowledge of resonance production, by significantly varying the parameters of our simulation. Results indicate that the uncertainties are well within the tentative error bars 


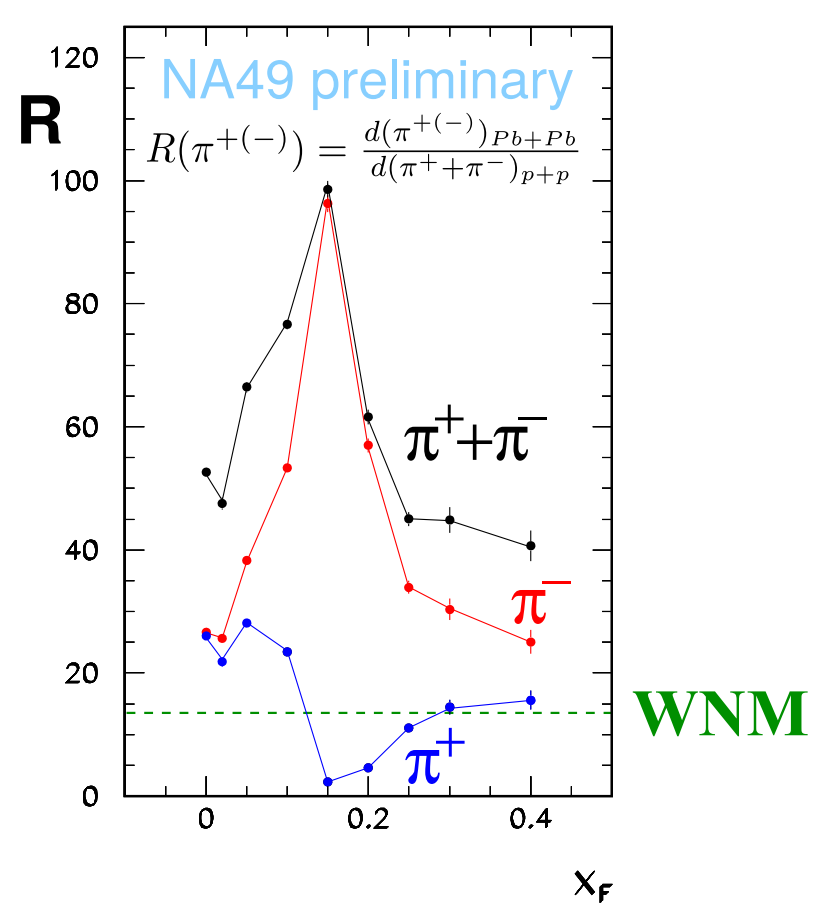

Figure 2. The ratios $\mathrm{R}\left(\pi^{+}\right)$of positive, $\mathrm{R}\left(\pi^{-}\right)$of negative and $\mathrm{R}\left(\pi^{+}+\pi^{-}\right)$of summed invariant particle densities for charged pions produced in $\mathrm{Pb}+\mathrm{Pb}$ peripheral versus $\mathrm{p}+\mathrm{p}$ collisions in the NA49 experiment, as a function of $x_{F}$. The dashed line indicates the Wounded Nucleon Model prediction for $R\left(\pi^{+}\right)=R\left(\pi^{-}\right)$, neglecting isospin effects.

associated with our present $d_{E}$ estimations, and can further be reduced by future experimental and theoretical investigation. We therefore expect that the study of EM effects can improve our knowledge of the space-time evolution of the system up to very high rapidities (where the latter is apparently least known).

At the present moment, the space-time characteristics of this system, tentatively established from these effects in the collision energy range $\sqrt{s_{N N}}=7.7-17.3 \mathrm{GeV}$ discussed here and shown in Figs 5(d) and 5(c), can give an independent estimate for the time of pion emission as a function of its rapidity. At midrapidity this time being equal to $d_{E}\left(y / y_{\text {beam }}=0\right) / \beta$, where $\beta$ is the spectator velocity, our preliminary estimate gives $5.3 \pm 2.2 \mathrm{fm} / \mathrm{c}$ for the time of pion emission in this region. For comparison, the pion decoupling times compiled in the published HBT analysis by the ALICE Collaboration [10] would indicate a value of approximately $\tau_{f} \approx 6 \mathrm{fm} / \mathrm{c}$ for the same range of collision energies.

\section{Conclusions and outlook}

On the basis of the above analysis, we can conclude that spectator-induced EM interactions can serve as a useful tool for studying the space-time evolution of heavy-ion collision systems, and in particular the longitudinal evolution from "hot" QGP matter at $y \approx 0$ to "cold" hadronic/nuclear matter at $y \approx$ $y_{\text {beam }}$. Our MC simulations of the production and decay of baryonic and mesonic resonances indicate 


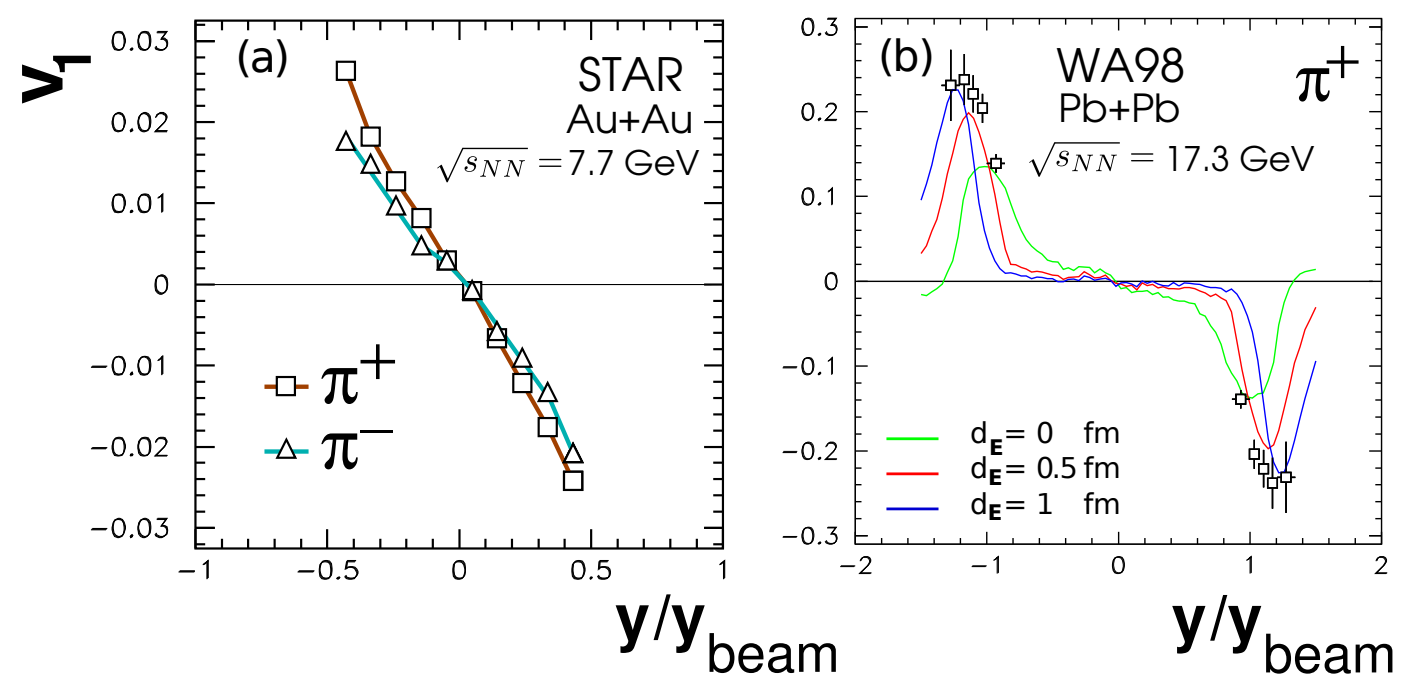

Figure 3. Directed flow $v_{1}$ of $\pi^{+}, \pi^{-}$as a function of the ratio of pion to beam rapidity in the c.m.s., $y / y_{\text {beam }}$, for (a) $\mathrm{Au}+\mathrm{Au}$ collisions at $\sqrt{s_{N N}}=7.7 \mathrm{GeV}$ (STAR Collaboration [5]), plot from [6] (see also [4]), and (b) $\mathrm{Pb}+\mathrm{Pb}$ collisions at $\sqrt{s_{N N}}=17.3 \mathrm{GeV}$ (WA98 Collaboration [7]), plot from [2].

that the space-time evolution of pion production at $y=y_{\text {beam }}$, i.e. non-zero $d_{E}$, can be understood as resulting from the space-time evolution of resonance decay. Further analysis of new experimental data on EM effects, on flow as well as $\pi^{+} / \pi^{-}$ratios, augmented by MC simulations, would improve our phenomenological knowledge and reduce the estimated uncertainties of the dependence of $d_{E}$ on pion rapidity $y / y_{\text {beam }}$, and possibly $p_{T}$. What is needed is precise data on the dependence of EM effects on such variables as collision geometry, reaction type, centrality, energy, particle type, rapidity and $p_{T}$.

To this end, we are actively seeking to further our analysis and MC simulation on EM spectator effects as members of the NA61/SHINE Collaboration [11], a heavy ion fixed target collision experiment and the successor to the NA49 experiment. The NA61/SHINE scan program includes collisions of heavy and intermediate size nuclei in the $\sqrt{s_{N N}}=6.3-16.8 \mathrm{GeV}$ energy range; a systematic analysis of EM effects on non-central collision data would cast new light on the behavior of $d_{E}$ with pion rapidity and transverse momentum, and hence on the space-time evolution of heavy ion collisions.

Acknowledgments: This work was supported by the National Science Centre, Poland (grant no. 2014/14/E/ST2/00018).

\section{References}

[1] A. Rybicki, A. Szczurek, Phys. Rev. C 75, 054903 (2007)

[2] A. Rybicki and A. Szczurek, Phys. Rev. C 87, 054909 (2013)

[3] A. Rybicki, A. Szczurek, M. Klusek-Gawenda, N. Davis, V. Ozvenchuk and M. Kielbowicz, to appear in EPJ Web of Conf.

[4] A. Rybicki, A. Szczurek and M. Kłusek-Gawenda, Acta Phys. Polon. B 46, no. 3, 737 (2015), and references therein

[5] STAR Collab., L. Adamczyk et al., Phys. Rev. Lett. 112, 162301 (2014) 
[6] A. Rybicki, A. Szczurek and M. Klusek-Gawenda, EPJ Web Conf. 81 (2014) 05024. doi:10.1051/epjconf/20148105024

[7] WA98 Collab., H. Schlagheck, Nucl. Phys. A 663, 725 (2000)

[8] A. Rybicki, Acta Phys. Polon. B42, 867 (2011).

[9] A. Rybicki, A. Szczurek, M. Kiełbowicz, N. Davis and V. Ozvenchuk, Acta Phys. Polon. Supp. 9, 303 (2016), and references therein

[10] ALICE Collab., K. Aamodt et al., Phys. Lett. B 696, 328 (2011)

[11] NA61 Collab., N. Abgrall et al., CERN-SPSC-2015-038; SPSC-P-330-ADD-8
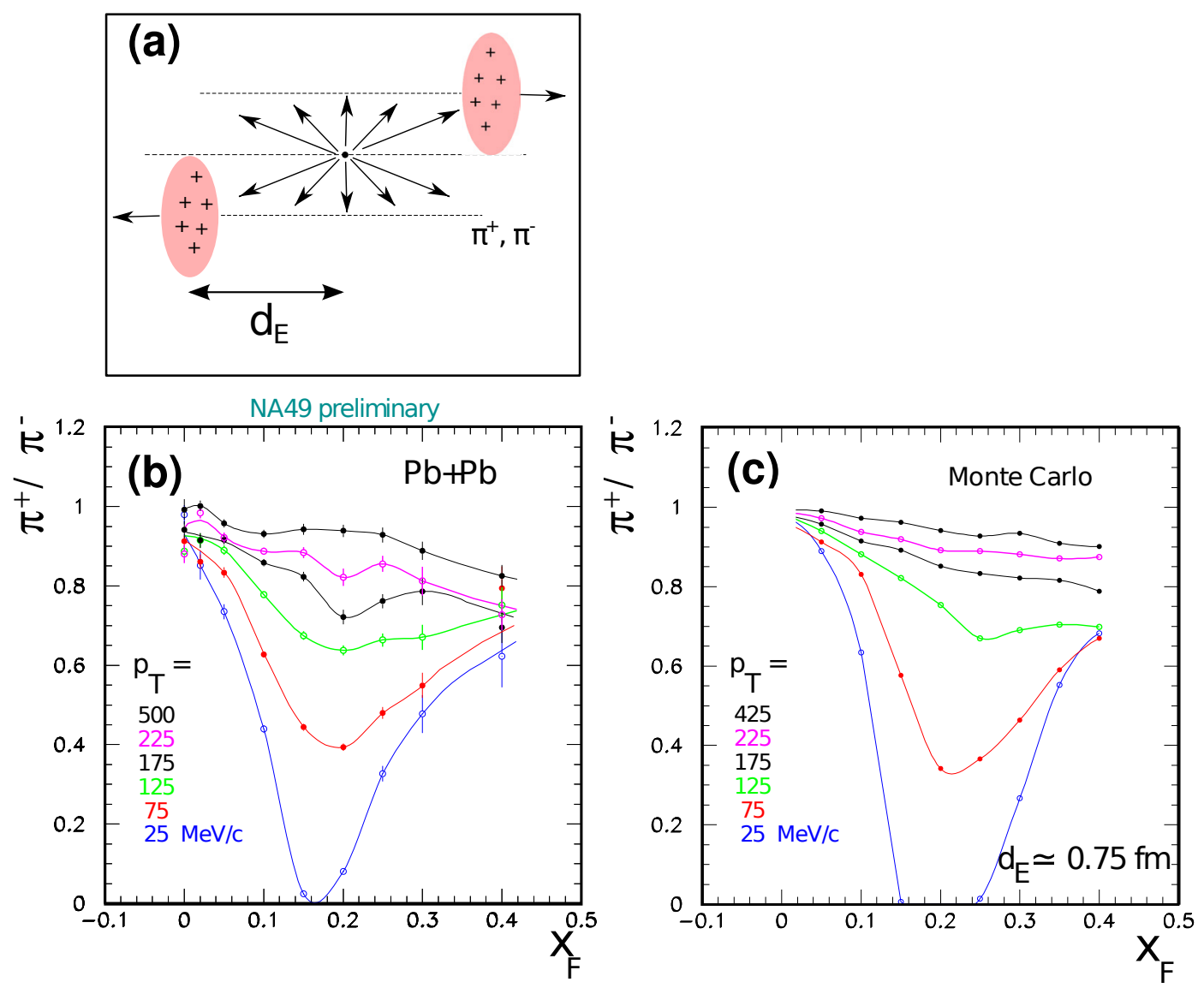

Figure 4. (a) Schematic representation of one-point emission model for spectator EM effects. (b) ratio of $\pi^{+}$over $\pi^{-}$distributions as a function of $x_{F}$ for various $p_{T}$ values, for $\mathrm{Pb}+\mathrm{Pb}$ collisions recorded in the NA49 experiment at $\sqrt{s_{N N}}=17.3 \mathrm{GeV}$. (c) ratio of $\pi^{+}$over $\pi^{-}$distributions for pions simulated by one-point emission model MC, for $d_{E} \approx 0.75 \mathrm{fm}$. Panels (b), (c) originally from [8]. 

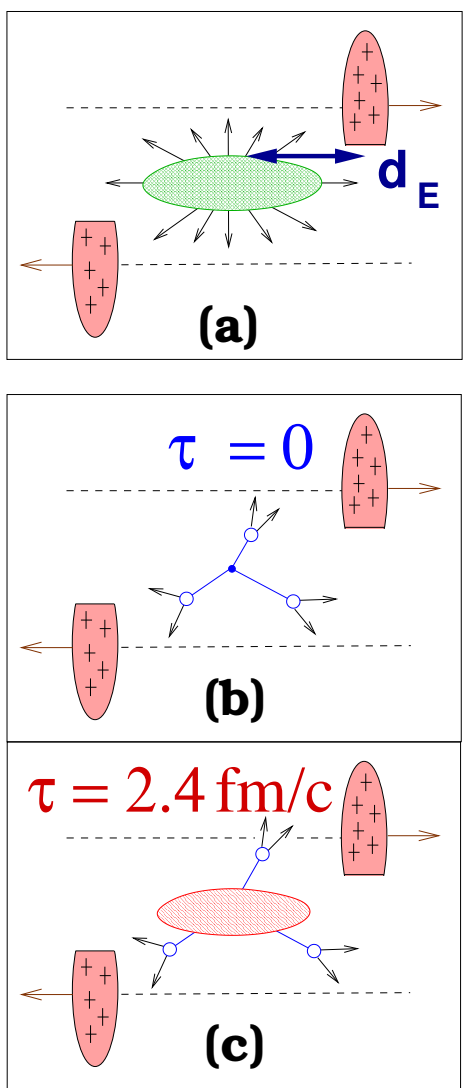
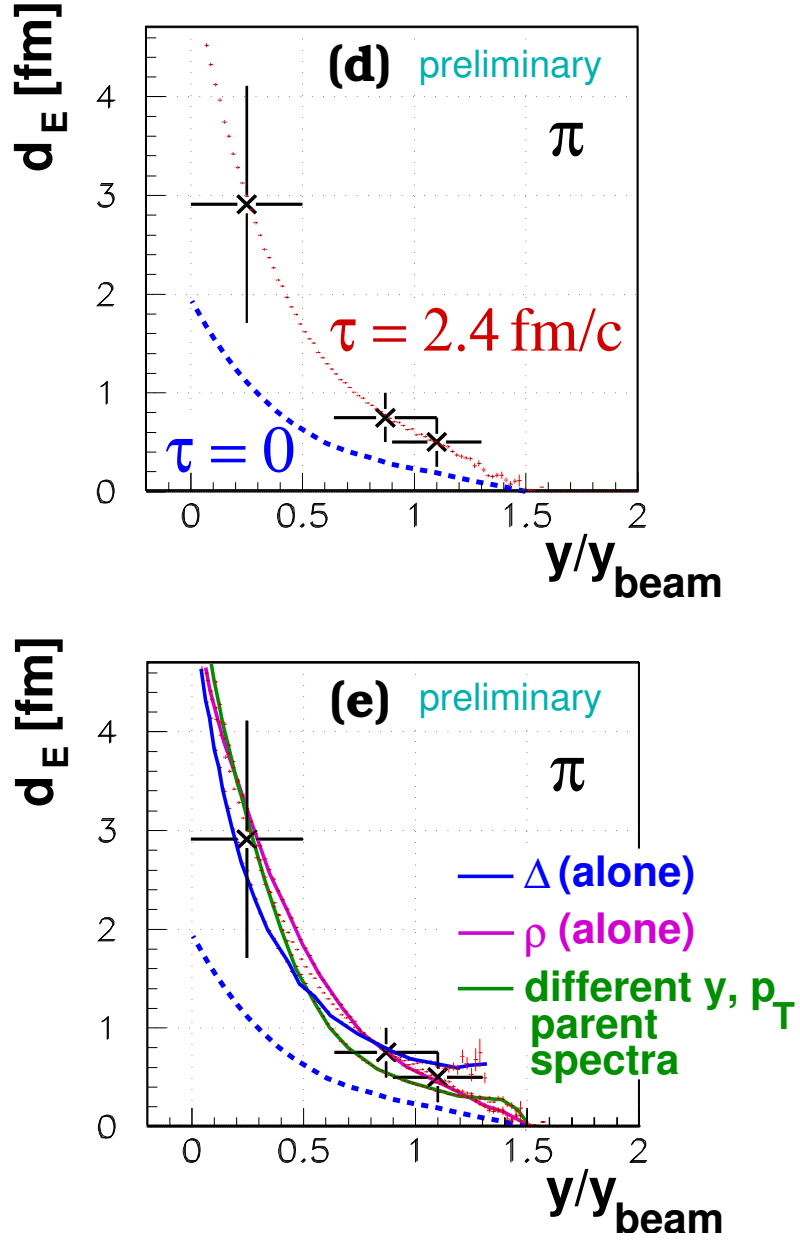

Figure 5. Information obtained on the basis of EM effects described in section 2: (a) schematic definition of $d_{E}$, (b) simulation of resonances produced instantly at the moment of the collision $(\tau=0)$ and (c) produced from an intermediate system characterized by a given proper lifetime $\tau$, (d) dependence of $d_{E}$ on pion rapidity, deduced from EM effects (data points), compared to the results of our Monte Carlo simulation illustrated in panels (b) and (c), respectively. (e) uncertainty of the present analysis induced by our incomplete knowledge of resonance production, estimated by assuming different characteristics for the input mix of parent $\Delta, \rho$ resonances. All the panels originate from [9]. 